Conclusions: Interhemispheric connectivity between the auditory cortices permits the association of basic auditory information with higher order language processing as the left and right auditory cortices are primarily responsible for different linguistic functions. Without efficient integration of auditory and linguistic experiences, errors in the interpretation of auditory phenomenon may occur; thus, this failure to integrate may result in the experience of AHs.

\section{Responding to deliberate self- harm among school students: the development and evaluation of evidence-based training}

\section{S Gook, J Robinson, A Yung, P McGorry, HP Yuen, T Jorm}

ORYGEN Research Centre, Melbourne, Australia

Background: Deliberate self-harm is prevalent among school students; however, evidence-based training on responding to this is limited.

Aims: The aims of the study were to develop and evaluate a training package for school staff to assist them manage self-harm among young people.

Methods: The training was a 2-day evidence-based package specifically designed for school staff. It aimed to better enable participants to understand and recognize self-harm and mental illness; improve the levels of confidence and perceived skill of participants in their ability to identify and manage self-harm, mental illness and risk and to improve attitudes of participants toward young people engaging in self-harm. The evaluation adopted a pretest/posttest design. Baseline measures were administered immediately prior to the training and were repeated immediately after the training. They will also be repeated at 6-month follow-up.

Results/Conclusions: The training was delivered to 213 school welfare staff across Victoria between May and August. Part 1 of the evaluation is now complete and analyses are under way. The findings will be presented and the overall efficacy of the training will be discussed.

\section{Increasing clinical monitoring of metabolic health in patients treated with antipsychotic medication}

\section{Graydon ${ }^{1,2}$, J Kulkarni', A deCastella ${ }^{1}$}

'Alfred Psychiatry Research Centre, The Alfred and Monash University, School of Psychology, Psychiatry \& Psychological Medicine, Melbourne, Australia; and ${ }^{2}$ Murdoch University, Perth, Australia
A priority in the treatment of schizophrenia and related conditions is to bring symptoms such as psychosis under control. To date, the most effective treatment modality to achieve this goal is pharmacotherapy. Unfortunately, a common side-effect of antipsychotic medication is weight gain, which in turn is linked to other serious physical conditions such as diabetes. The metabolic side-effects of taking antipsychotics can so impact upon the patient that they choose to skip or discontinue taking medication, which puts them at increased risk of relapse. Thus, improving the general health of people with schizophrenia and controlling the side-effects of antipsychotic medication are important clinical goals. The monitoring of patients' weight, body mass index, lipids and blood pressure is an important component of maintaining physical health and is an important factor in maintaining adherence to medication regimes. If metabolic side-effects do develop, use of a monitoring instrument would alert the clinician to the fact. We present an overview of the issues around monitoring of metabolic sideeffects, as well as an overview of a study currently being conducted, which assesses clinician's monitoring practices. The primary aim of this study was to assess metabolic monitoring practices by clinicians with a range of experience. A set of metabolic monitoring guidelines will be implemented, and the same clinicians will be reassessed over a 6-month period. This research will result in more consistent monitoring of metabolic side-effects, leading to improved mental and physical health outcomes for patients receiving antipsychotic medications.

\section{Perceived burden in carers of patients with mild Alzheimer's disease: the effect of patient insight}

\author{
K Greenop, 0 Almeida, K Cox, L Flicker, J Foster, \\ S Starkstein, N Lautenschlager
}

University of Western Australia, Perth, Australia

Background: Caring for a family member with dementia can cause stress; possible causes are the patient's lack of insight, poor functional ability or poor health. This study examined the association between level of insight in the patient and perceived burden in the carer.

Methods: Twenty-four patients with mild Alzheimer's disease and their carers were recruited from Perth Metropolitan Memory clinics and community advertising. Patients were administered a neuropsychological battery and completed the Geriatric Depression Scale (GDS), Dysexecutive (DEX) questionnaire and SF-36 health survey. Carers were interviewed separately on 